\title{
Mortality and the Risk Factors in Elderly Female Patients With Femoral Neck and Trochanteric Fractures
}

\author{
Toshihiro Higashikawa a, b, h, Kenji Shigemoto ${ }^{\mathrm{c}}$, Kenichi Goshima ${ }^{\mathrm{c}}$, Takeshi Horiic ${ }^{\mathrm{c}}$, Daisuke Usuda ${ }^{\mathrm{d}}$, \\ Takuro Morita $^{\mathrm{b}}$, Manabu Moriyama ${ }^{\mathrm{e}}$, Hiromi Inujima ${ }^{\mathrm{f}}$, Masahiro Hangyou ${ }^{\mathrm{f}}$, Kimiko Usuda ${ }^{\mathrm{f}}$, \\ Shigeto Morimoto ${ }^{\mathrm{b}}$, Tadami Matsumotog ${ }^{\mathrm{g}}$, Shigeki Takashima ${ }^{\mathrm{d}}$, Tsugiyasu Kanda ${ }^{\mathrm{d}}$, \\ Masashi Okuro ${ }^{\mathrm{b}}$, Takeshi Sawaguchic
}

\begin{abstract}
Background: The main purpose of this study is to exhaustively explore risk factors, including age, gender, and several clinical indices, for mortality in elderly patients with femoral neck fracture and to evaluate some of them using survival analyses.
\end{abstract}

Methods: This was a retrospective study tracking 1 year for vital prognosis. Data were collected at post-operation from medical records of the cases. Survival analysis was conducted to investigate the risk factors for death, including albumin, urinary retention, activity of daily living (ADL), and cognitive disorder.

Results: We recruited 318 patients with a history of hip surgery carried out at Toyama Municipal Hospital, in which 39 patients died for 1 year after discharge. The results showed a significant decrease in survival rate in low albumin, positive urinary retention, and low $\mathrm{ADL}(\mathrm{P}<0.01$, by log-rank test). The hazard ratios (95\% confidence interval) of albumin, urinary retention, ADL, and cognitive disorder were 0.36 (0.19 - 0.69), 0.4 (0.2 - 0.8), $0.29(0.15-0.58)$ and $0.65(0.32-1.29)$, respectively.

Conclusions: This study demonstrated that albumin, urinary retention

Manuscript submitted July 24, 2020, accepted August 13, 2020

Published online September 21, 2020

${ }^{a}$ Department of Geriatric Medicine, Kanazawa Medical University Himi Municipal Hospital, 1130, Kurakawa, Himi, Toyama 935-8531, Japan

bDepartment of Geriatric Medicine, Kanazawa Medical University, Uchinada, Kahoku-gun, Ishikawa 920-0293, Japan

'Department of Orthopedics and Joint Reconstructive Surgery, Toyama Municipal Hospital, Hokubumachi, Imaizumi, Toyama, Toyama 939-8511, Japan ${ }^{d}$ Kanazawa Medical University, Uchinada, Kahoku-gun, Ishikawa 920-0293, Japan

eDepartment of Urology, Kanazawa Medical University Himi Municipal Hospital, Kurakawa, Himi, Toyama 935-8531, Japan

${ }^{f}$ Toyama Municipal Hospital, Hokubumachi, Imaizumi, Toyama, Toyama 9398511, Japan

'Department of Orthopedic Medicine, Kanazawa Medical University, Uchinada, Kahoku-gun, Ishikawa 920-0293, Japan

${ }^{\mathrm{h}}$ Corresponding Author: Toshihiro Higashikawa, Department of Geriatric Medicine, Kanazawa Medical University Himi Municipal Hospital, 1130, Kuragawa, Himi, Toyama 935-8531, Japan. Email: himikmu@yahoo.co.jp

doi: https://doi.org/10.14740/jocmr4292 and ADL were the important risk factors for mortality, and suggested that the postoperative management of albumin, urinary retention and $\mathrm{ADL}$ is important, especially in elderly female patients receiving surgery of femoral neck and trochanteric fractures.

Keywords: Elderly; Hip fractures; Albumin; Urinary retention; Activity of daily living; Cognitive disorder

\section{Introduction}

A nationwide survey of hip fractures by the Japanese Orthopedic Association (JOA) from 1998 to 2008 found that a drastic increase occurred in the number of patients with hip fractures with time in Japan [1]. Toyama City is one of the large cities in Japan, and the elderly population exceeds $30 \%$ of the total population.

Among them, Toyama City Hospital carries out approximately 150 operations per year for femoral neck fracture, and it is increasing every year. Hip fractures in older adults are a leading public health concern, although the key factor for mortality is still controversial [2]. Proximal femoral fractures significantly reduce activity of daily living (ADL) in patients with numerous perioperative complications. It is of paramount importance to conduct multi-occupation cooperation to manage the patients as a whole, by cooperating with physicians, pharmacists, rehabilitation therapists, nurses, etc. Toyama City Hospital has, for the first time in Japan, enabled not only orthopedic surgeons but also physicians to perform general examinations on admission and consult specialists. On this situation, diagnostic criteria should be simple for efficient collaboration. Therefore, the perioperative management by the multi-occupation cooperation is carried out without being influenced by individual ability, and the orthopedic ward with the specialized staff specialized for the femoral proximal part fracture is included. The use of the multidisciplinary treatment approach led to a significant increase in osteoporosis treatment rate and better functional recovery [3].

A prospective study showed that $10.7 \%$ of the patients developed urinary retention after operation [4]. A study investigating the 1-year mortality of patients treated in a hip fracture 
program for elders has found that ADL dependence was a borderline predictor for mortality [2]. A population-based study has also found that cognitive disorder is an independent predictor of mortality following surgery for fractures of the hip [5].

The present study aimed to investigate association of albumin, urinary retention, ADL and cognitive disorder with mortality in patients with hip fracture through vital prognosis tracking for 1 year.

\section{Patients and Methods}

This retrospective study was carried out under the approval of the Clinical Research Ethics Committee of Toyama Municipal Hospital. The study was conducted in accordance with the guidelines of the Declaration of Helsinki. Written informed consent was obtained from all patients. Data were collected at post-operation. The inclusion criteria were that patients who were at the age of $\geq 65$ years, and who underwent surgery of femoral neck and trochanteric fracture in our institute. The exclusion criteria were that the patients unable to follow-up during the observation period, or patients with no measurement of serum albumin. Demographic information, including age and gender, was collected by physicians, specialist nurses, pharmacists, and medical affairs. The existence of the urinary retention was also assessed in the same division. We followed up the cases every 3 months, up to 12 months.

Serum albumin concentration was measured as a standard test package of blood tests at admission, and the value itself was used for the following statistical analysis as continuous variables. In addition, the data were divided into high albumin $(\geq 3.5 \mathrm{~g} / \mathrm{dL})$ and low albumin $(<3.5 \mathrm{~g} / \mathrm{dL})$ groups that were used for the following analysis as dichotomous variables in the survival analysis.

In all cases, urethral catheter was inserted pre-operatively according to a urinary retention manual originally created in our hospital. These methods were conducted in parallel, regardless of the subjectivity of physicians. The urinary retention was defined as a state of difficulty in micturition under storage of $400-500 \mathrm{~mL}$ of urine in a bladder after removal of the catheter, symptoms of dysuria or decreases in micturition desire under $>100 \mathrm{~mL}$ of residual urine. The cases were defined as urinary retention and others were defined as "no urinary retention" group.

Degree of independence in everyday life on each case was assessed by a conventional ADL scale, which is classified by the four groups (J, A, B, C). Group J is mostly assistancefree, group A is semi-bedridden mostly active during daytime, group $\mathrm{B}$ is bedridden requiring assistance for daily activities, and group $\mathrm{C}$ is bedridden requiring assistance in bed [6]. We divided them into two groups: JA (assistance non-required) and $\mathrm{BC}$ (assistance-required) groups. JA group was regarded as "good ADL" group.

Cognitive disorders were diagnosed by neuropsychiatrists based on the current Diagnostic and Statistical Manual of Mental Disorders (DSM-V). Data were collected from medical records of the cases. We also regarded absence of cognitive disorder group as "no cognitive disorder" group.

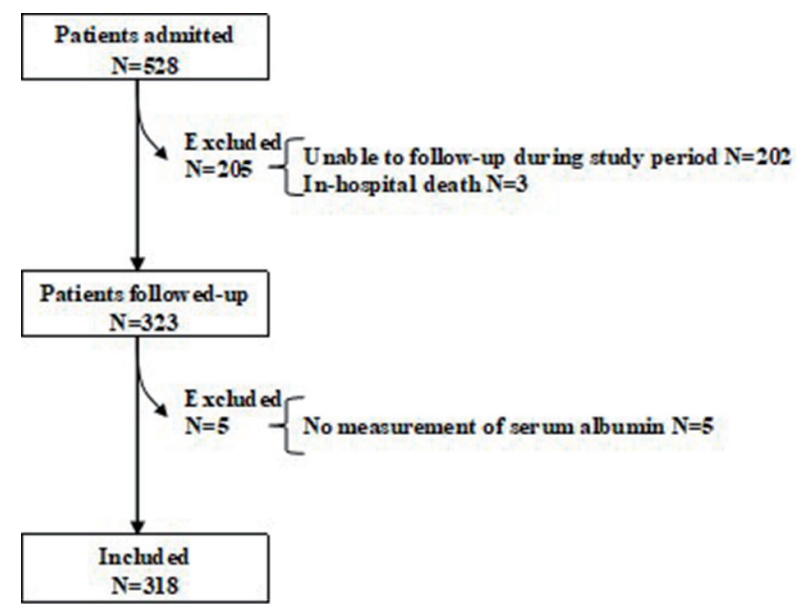

Figure 1. Flow chart of the study.

The present study includes continuous data, such as age, body mass index, serum albumin concentration, waiting time, and hemoglobin, which were statistically evaluated by mean, standard deviation and Student $t$-test.

The present study also consists of dichotomous data, such as gender, urinary retention, $\mathrm{ADL}$, cognitive disorder, fracture site, diabetes mellitus, hypertension, cardiovascular disorder, kidney disorder and bone fracture, which were statistically evaluated by Chi-square test.

Survival analyses were performed using albumin, urinary retention, ADL and cognitive disorder as the factors described as Kaplan-Meier plot, and the statistical differences of the two different survival curves were evaluated by log-rank test. Ageand sex-adjusted Cox regression analyses were also performed to evaluate hazard ratios for each factor.

All statistical analyses were two-tailed, $\mathrm{P}<0.05$ was regarded as significant, and Student $t$-test, Chi-square test and survival analyses were performed using Easy R (EZR , Saitama Medical Center, Jichi Medical University, Saitama, Japan) [7].

\section{Results}

Initially 528 patients were admitted to the Toyama Municipal Hospital from January 1, 2016 to December 31, 2017, excluding 205 patients including those unable to follow-up during observation period $(n=202)$ and lack of albumin data $(n=5)$. Finally, 318 patients were used as an analysis set of the present study, as depicted in Figure 1.

Table 1 shows demographics of the patient background at the enrollment in this study. For the patient population, the information was $\mathrm{n}=318$, men/women: $67 / 251,81.94 \pm$ $7.36 / 84.03 \pm 7.21$ years of age. The population was divided into survived $(\mathrm{n}=279)$ and dead $(\mathrm{n}=39)$ groups. Fracture site, waiting time, cognitive disorder, diabetes mellitus, hypertension, cardiovascular diseases, kidney disorder, and bone fracture showed no significant differences between the groups. On the other hand, gender, age, serum albumin, urinary retention, ADL, and hemoglobin showed significant differences between the groups $(\mathrm{P}<0.01,<0.015,<0.01,<0.01,<0.01,<0.01$, 
Table 1. Demographics of Patient Background

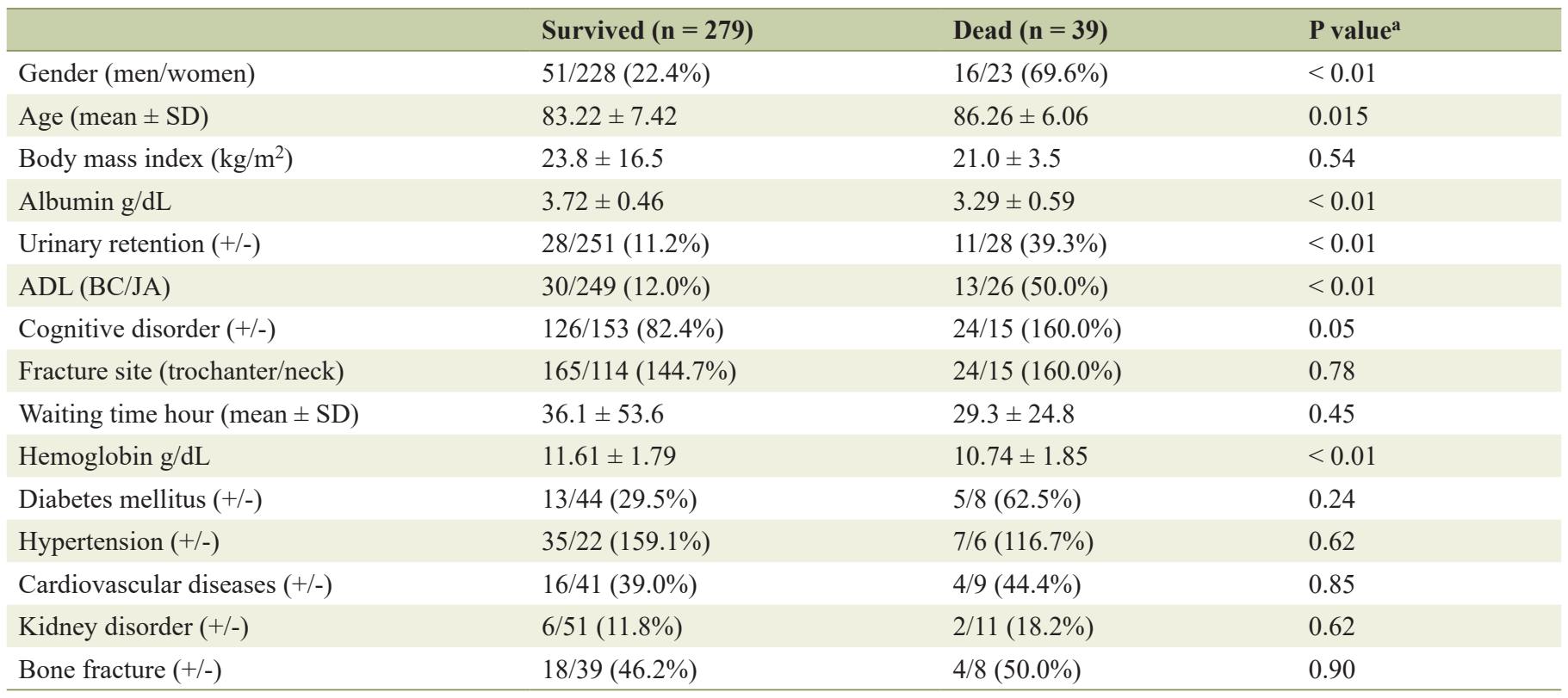

at-test (age, BMI, albumin, hemoglobin, waiting time hour), Chi-square test (others). ADL: activity of daily living; BMI: body mass index; SD: standard deviation.

respectively).

Figure 2 shows Kaplan-Meier plots indicating that albumin (high/low), urinary retention (negative/positive), and ADL (JA/BC) showed significant differences $(\mathrm{P}<0.01$, by log-rank test). Cognitive disorder (negative/positive) showed a borderline significant difference ( $\mathrm{P}=0.05$, by log-rank test).

Table 2 shows the Cox regression analysis indicating that albumin (hazard ratio referred to low albumin: $0.36,95 \%$ confidence interval (CI) $0.19-0.69, \mathrm{P}<0.01$ ) as well as urinary retention (hazard ratio referred to positive urinary retention: $0.39,95 \%$ CI $0.19-0.79, \mathrm{P}<0.01$ ) and ADL (hazard ratio referred to $\mathrm{BC}, 0.29,95 \% \mathrm{CI} 0.15-0.58, \mathrm{P}<0.01)$ were significant hazard ratio under adjustment of age and sex as covariates. Cognitive disorder showed no significant difference.

\section{Discussion}

The present study revealed that adjusted patient survival was significantly higher in patients with higher serum albumin concentration $(\geq 3.5 \mathrm{~g} / \mathrm{dL})$, non-urinary retention, and higher $\mathrm{ADL}(\mathrm{J}$ or $\mathrm{A})$. The overall adjusted hazard ratio in high serum albumin, non-urinary retention and high ADL groups were approximately 0.4 , compared to other groups. On the other hand, the adjusted hazard ratio in patients in non-cognitive disorder group was 0.65 , which was not significant compared to cognitive-disorder group, although log-rank test showed borderline significance $(\mathrm{P}=0.05)$. Hemoglobin, estimated glomerular filtration rate (eGFR), respiratory disorder, and pneumonia were also suggested to be underlying risk factors as shown in Table 1, although we did not conduct detailed investigation.

Serum albumin level has been known to be the most well- established serum marker of malnutrition. A retrospective cohort study of geriatric patients undergoing a hip fracture surgical procedure in USA showed that hypoalbuminemia is a powerful independent risk factor for mortality following a surgical procedure for geriatric hip fracture, which suggests that further investigation into postoperative nutritional supplementation is warranted to decrease the risk of complications [8]. Malnutrition itself was also associated with an increase in mortality. Nutritional intervention was cost effective and was associated with an improvement in nutritional status and a greater functional recovery in older people; thus, the prevention of malnutrition and an early nutritional intervention can improve recovery following a hip fracture [9]. Perioperative urinary retention is also known to be highly prevalent in elderly hip fracture patients. It adversely affects the functional outcome of female patients but is not associated with increased mortality rates [10]. Our retrospective study has demonstrated that cognitive function as well as ADL were the important risk factors for urinary retention, suggesting that the postoperative management of urinary retention is important with taking account of neurofunctional assistance and nursing care in daily living, especially in elderly female patients receiving surgery of femoral neck and trochanteric fractures [11]. Complications after hip fracture surgery were common. Advanced age and delay in surgery were also associated with higher complication rates [12]. Satisfactory voiding resumes earlier with the use of intermittent catheterization, if begun at the onset of urinary retention and repeated at regular intervals, than with the use of an indwelling catheter in elderly patients who have undergone surgical repair of hip fractures [13]. Another study also showed that the early removal of the urethral catheter, and preoperative dementia and/or delirium had significant corre- 

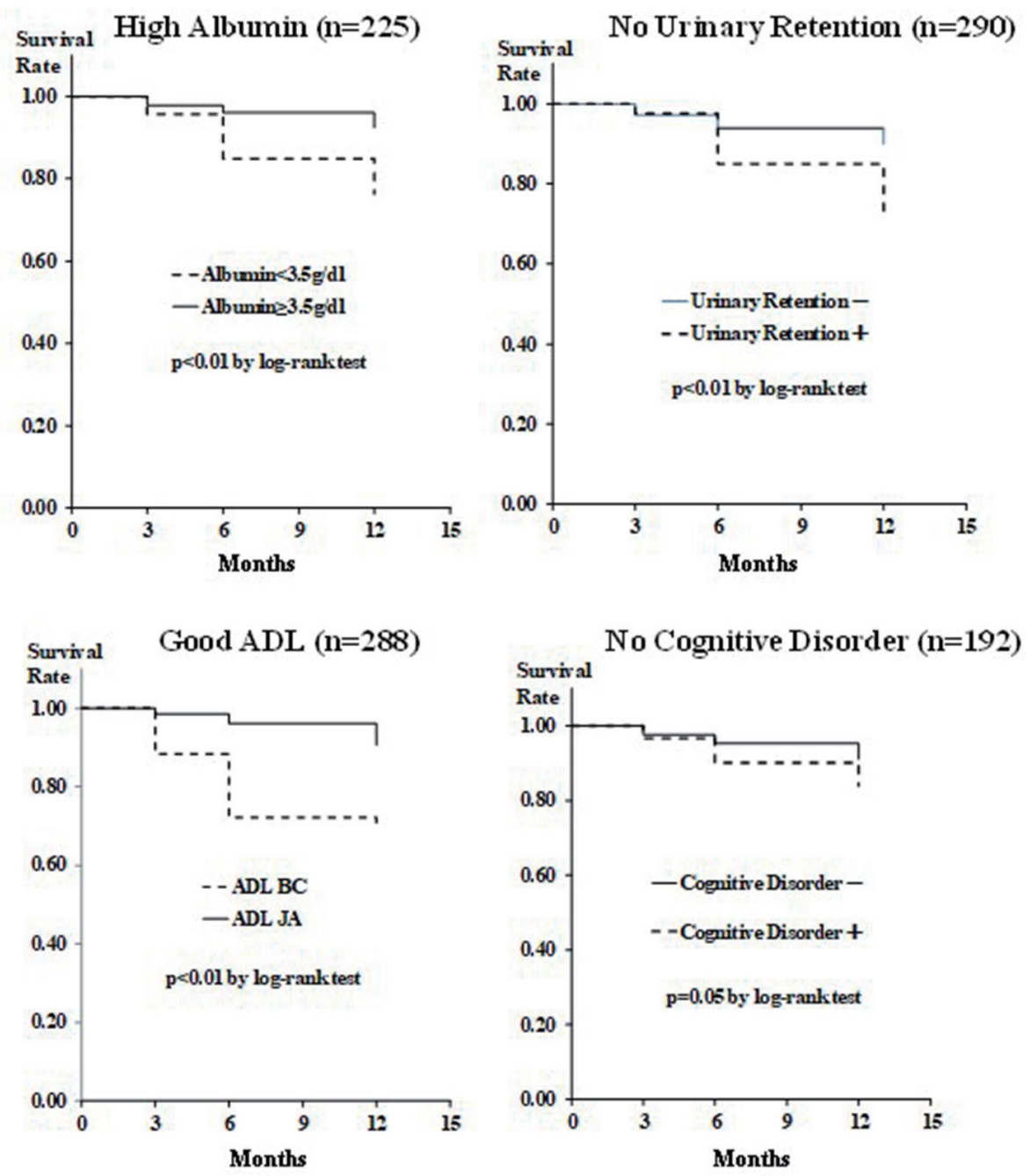

Figure 2. Kaplan-Mayer survival curves comparing albumin, urinary retention, ADL and cognitive disorder. ADL: activity of daily living.

lations with postoperative urinary retention [14]. Under these circumstances, incorporation of the cooperative management of patients by geriatricians or hospitalists and orthopedists has been progressing in a worldwide scale. Evidence-based treatment protocols for the care of elderly patients have been applied to patients with hip fractures [15-21].

The current study has several limitations. First, the present results have little sample size, especially in dead patient group $(n=39)$. This should be taken into consideration on the interpretation of the present results. Second, the present study population included male/female patients, but the mechanism of urinary retention is different between genders. Third, there could exist many risk factors for mortality that should be taken into consideration, such as waiting time, hemoglobin, respiratory functions, etc., which are the factors relevant to mortality. Fourth, this study does not include healthy elderly control; thus, the causal relationship between mortality and the risk factors is not clear enough. It is desirable to investigate the

Table 2. Hazard Ratios Calculated by Age- and Sex-Adjusted Cox Regression Analyses

\begin{tabular}{lllll} 
& Hazard ratio & Lower 95\% CI & Upper 95\% CI & P value \\
\hline High albumin & 0.36 & 0.19 & 0.69 & $<0.01$ \\
Urinary retention (-) & 0.39 & 0.19 & 0.79 & $<0.01$ \\
Good ADL & 0.29 & 0.15 & 0.58 & $<0.01$ \\
Cognitive disorder (-) & 0.65 & 0.32 & 1.29 & 0.22 \\
\hline
\end{tabular}

$\mathrm{Cl}$ : confidence interval; ADL: activity of daily living. 
cause of death after discharge. It is also necessary to build a framework for regional inclusion in the future. Further investigation will be required to comprehensively evaluate risk of mortality and to elucidate key factors relevant to the prognosis of postoperative femoral fracture.

\section{Conclusions}

In conclusion, our analysis of 318 elderly patients with femoral neck and trochanteric fractures suggests that low serum albumin, positive urinary retention and low ADL are important risk factors for mortality. Further studies will be required to determine appropriate benchmarks for differing mortality risk profiles.

\section{Acknowledgments}

None to declare.

\section{Financial Disclosure}

None to declare.

\section{Conflict of Interest}

None to declare.

\section{Informed Consent}

Informed consents were obtained from all participants.

\section{Author Contributions}

T Higashikawa and KS have made data curation; T Higashikawa, KG, T Horii, DU, T Morita and MM designed the research. T Higashikawa conducted data analysis and acquired funding. T Higashikawa, $\mathrm{HI}, \mathrm{MH}$, and $\mathrm{KU}$ conducted investigation of medical records. SM, T Matsumoto, ST, TK, MO, and TS supervised this research. T Higashikawa wrote original draft of this manuscript. All authors read and approved the final manuscript.

\section{Data Availability}

The authors declare that data supporting the findings of this study are available within the article.

\section{References}

1. Hagino H, Endo N, Harada A, Iwamoto J, Mashiba T,
Mori S, Ohtori S, et al. Survey of hip fractures in Japan: Recent trends in prevalence and treatment. J Orthop Sci. 2017;22(5):909-914.

2. Schnell S, Friedman SM, Mendelson DA, Bingham KW, Kates SL. The 1-year mortality of patients treated in a hip fracture program for elders. Geriatr Orthop Surg Rehabil. 2010;1(1):6-14.

3. Shigemoto K, Sawaguchi T, Goshima K, Iwai S, Nakanishi A, Ueoka K. The effect of a multidisciplinary approach on geriatric hip fractures in Japan. J Orthop Sci. 2019;24(2):280-285.

4. Waterhouse N, Beaumont AR, Murray K, Staniforth P, Stone $\mathrm{MH}$. Urinary retention after total hip replacement. A prospective study. J Bone Joint Surg Br. 1987;69(1):6466.

5. Chiu HC, Chen CM, Su TY, Chen CH, Hsieh HM, Hsieh $\mathrm{CP}$, Shen DL. Dementia predicted one-year mortality for patients with first hip fracture: a population-based study. Bone Joint J. 2018;100-B(9):1220-1226.

6. Kobayashi Y, Yuzawa K, Hotta F, Usuda D, Okuro M, Moriyama M, et al. Rehabilitation liaison from convalent to maintenance stage in Cooperation with a Municipal Medical Association and Administration: an attempt in Sumida City, Tokyo. JMAJ. 2010;53(24):294-300.

7. Kanda Y. Investigation of the freely available easy-touse software 'EZR' for medical statistics. Bone Marrow Transplant. 2013;48(3):452-458.

8. Bohl DD, Shen MR, Hannon CP, Fillingham YA, Darrith B, Della Valle CJ. Serum albumin predicts survival and postoperative course following surgery for geriatric hip fracture. J Bone Joint Surg Am. 2017;99(24):2110-2118.

9. Malafarina V, Reginster JY, Cabrerizo S, Bruyere O, Kanis JA, Martinez JA, Zulet MA. Nutritional status and nutritional treatment are related to outcomes and mortality in older adults with hip fracture. Nutrients. 2018;10(5).

10. Adunsky A, Nenaydenko O, Koren-Morag N, Puritz L, Fleissig Y, Arad M. Perioperative urinary retention, shortterm functional outcome and mortality rates of elderly hip fracture patients. Geriatr Gerontol Int. 2015;15(1):65-71.

11. Higashikawa T, Shigemoto K, Goshima K, Usuda D, Okuro M, Moriyama M, Inujima H, et al. Urinary retention as a postoperative complication associated with functional decline in elderly female patients with femoral neck and trochanteric fractures: A retrospective study of a patient cohort. Medicine (Baltimore). 2019;98(24):e16023.

12. Poh KS, Lingaraj K. Complications and their risk factors following hip fracture surgery. J Orthop Surg (Hong Kong). 2013;21(2):154-157.

13. Skelly JM, Guyatt GH, Kalbfleisch R, Singer J, Winter L. Management of urinary retention after surgical repair of hip fracture. CMAJ. 1992;146(7):1185-1189.

14. Tobu S, Noguchi M, Hashikawa T, Uozumi J. Risk factors of postoperative urinary retention after hip surgery for femoral neck fracture in elderly women. Geriatr Gerontol Int. 2014;14(3):636-639.

15. Fisher AA, Davis MW, Rubenach SE, Sivakumaran S, Smith PN, Budge MM. Outcomes for older patients with hip fractures: the impact of orthopedic and geriatric medicine cocare. J Orthop Trauma. 2006;20(3):172-178; dis- 
cussion 179-180.

16. Naglie G, Tansey C, Kirkland JL, Ogilvie-Harris DJ, Detsky AS, Etchells E, Tomlinson G, et al. Interdisciplinary inpatient care for elderly people with hip fracture: a randomized controlled trial. CMAJ. 2002;167(1):25-32.

17. Roberts HC, Pickering RM, Onslow E, Clancy M, Powell J, Roberts A, Hughes K, et al. The effectiveness of implementing a care pathway for femoral neck fracture in older people: a prospective controlled before and after study. Age Ageing. 2004;33(2):178-184.

18. Thwaites JH, Mann F, Gilchrist N, Frampton C, Rothwell A, Sainsbury R. Shared care between geriatricians and orthopaedic surgeons as a model of care for older patients with hip fractures. N Z Med J. 2005;118(1214):U1438.

19. Koval KJ, Chen AL, Aharonoff GB, Egol KA, Zuckerman JD. Clinical pathway for hip fractures in the elderly: the hospital for joint diseases experience. Clin Orthop Relat Res. 2004;425:72-81.

20. Parker MJ, Pryor GA, Myles J. 11-year results in 2,846 patients of the Peterborough Hip Fracture Project: reduced morbidity, mortality and hospital stay. Acta Orthop Scand. 2000;71(1):34-38.

21. Vidan M, Serra JA, Moreno C, Riquelme G, Ortiz J. Efficacy of a comprehensive geriatric intervention in older patients hospitalized for hip fracture: a randomized, controlled trial. J Am Geriatr Soc. 2005;53(9):1476-1482. 